\title{
ON RIGIDITY PHENOMENA OF COMPACT SURFACES IN HOMOGENEOUS 3-MANIFOLDS
}

\author{
ZEJUN HU, DONGLIANG LYU, AND JING WANG
}

(Communicated by Lei $\mathrm{Ni}$ )

\begin{abstract}
Let $E(\kappa, \tau)$ be the 3-dimensional homogeneous Riemannian manifold with isometry group of dimension 4 , where $\kappa$ is the curvature of the basis and $\tau$ the bundle curvature, which satisfy $\kappa-4 \tau^{2} \neq 0$. A special case of $E(\kappa, \tau)$ is the Berger sphere that is also denoted by $\mathbb{S}_{b}^{3}(\kappa, \tau)$. In this paper, surfaces of $E(\kappa, \tau)$ are studied. As the main result, rigidity theorems in terms of the second fundamental form are established for compact (minimal) surfaces of $\mathbb{S}_{b}^{3}(\kappa, \tau)$.
\end{abstract}

\section{INTRODUCTION}

In recent years, surfaces in homogeneous Riemannian 3-manifolds have been extensively studied. See [1, 3, 9 11, 16, 19, among others. In this paper, we will study surfaces of the homogeneous Riemannian 3-manifolds whose isometry groups have dimension 4 .

Recall that the classification of simply connected 3-dimensional homogeneous Riemannian manifolds is well known (cf. [4,6, 12, 20]). Such a manifold has an isometry group of dimension 3,4 , or 6 . When the dimension of the isometry group is 6 , then we have the space form. When the dimension of the isometry group is 3 , the spaces are a certain class of Lie groups, among them we specially quote the space $\mathrm{Sol}_{3}$ which is the only manifold to be a "geometry model" in the sense of Thurston (see e.g. [13]). When the dimension of the isometry group is 4, such a manifold is a Riemannian fibration over a 2-dimensional space form, the fibers are geodesics and there exists a one-parameter family of translations along the fibers, generated by a unit Killing vector field $\xi$ which will be called the vertical vector field. These manifolds are classified, up to isometry, by the curvature $\kappa$ of the base surface of the fibration and the bundle curvature $\tau$, where $\kappa$ and $\tau$ can be any real numbers satisfying $\kappa \neq 4 \tau^{2}$.

The manifold whose isometry group is of dimension 4 as described above is denoted by $E(\kappa, \tau)$. Then there exists a Riemannian submersion $\Pi: E(\kappa, \tau) \rightarrow$ $\mathbb{M}^{2}(\kappa)$, where $\mathbb{M}^{2}(\kappa)$ is a 2-dimensional simply connected space form of constant curvature $\kappa$, with totally geodesic fibers and there exists a unit Killing vector field $\xi$ on $E(\kappa, \tau)$ which is vertical with respect to $\Pi$. The bundle curvature is the number

Received by the editors April 21, 2013 and, in revised form, August 3, 2013 and August 17, 2013.

2010 Mathematics Subject Classification. Primary 53C24; Secondary 53C20, 53C42.

Key words and phrases. Homogeneous 3-manifolds, Berger sphere, minimal surface, Hopf torus, Clifford torus.

This project was supported by grants of NSFC-11071225 and NSFC-11371330. 
$\tau$ such that $\bar{\nabla}_{X} \xi=\tau X \times \xi$ for any vector field $X$ on $E(\kappa, \tau)$, where $\times$ denotes the vector product and $\bar{\nabla}$ denotes the Riemannian connection of $E(\kappa, \tau)$, respectively. When $\tau=0$ (and then $\kappa \neq 0$ ), we get a product manifold $\mathbb{M}^{2}(\kappa) \times \mathbb{R}$, the vertical vector $\xi$ is tangent to the factor $\mathbb{R}$. This case was treated extensively; we refer to [8], among many others. The manifolds with $\tau \neq 0$ are of three types based on the value of $\kappa$ : the Berger spheres for $\kappa>0$ that is commonly denoted by $\mathbb{S}_{b}^{3}(\kappa, \tau)$; the Heisenberg group $\mathrm{Nil}_{3}$ for $\kappa=0$; and the universal cover $\widetilde{\mathrm{SL}}(2, \mathbb{R})$ of the Lie group $\mathrm{SL}(2, \mathbb{R})$ (endowed with a 2-parameter family of homogeneous metrics) for $\kappa<0$.

In this paper, we are concerned with the rigidity properties for surfaces of $E(\kappa, \tau)$ with $\tau \neq 0$ in terms of the second fundamental form. Compared with that which has been well-known for the space form, Theorem 1.1 shows that similar interesting rigidity phenomena still hold for compact minimal surfaces of the more general ambient space $E(\kappa, \tau)$.

To state our results, we recall the typical surfaces in $E(\kappa, \tau)$ which can be constructed in the following way. Given any regular curve $\gamma$ in $\mathbb{M}^{2}(\kappa), \Pi^{-1}(\gamma)$ is a surface in $E(\kappa, \tau)$ which has $\xi$ as a parallel tangent vector field and hence $\Pi^{-1}(\gamma)$ is a flat surface, usually we call $\Pi^{-1}(\gamma)$ a Hopf surface of $E(\kappa, \tau)$. If $\gamma$ is a closed curve, then we call $\Pi^{-1}(\gamma)$ a flat cylinder. Moreover, if $\gamma$ is a closed curve and $\Pi$ is a circle Riemannian submersion (this happens for $\mathbb{S}_{b}^{3}(\kappa, \tau)$ and $\widetilde{\mathrm{SL}}(2, \mathbb{R})$ ), then $\Pi^{-1}(\gamma)$ is a flat torus which is also called Hopf torus. A special case is $\left\{(z, w) \in \mathbb{S}_{b}^{3}(\kappa, \tau):|z|^{2}=|w|^{2}=\frac{1}{2}\right\}$ which is the well-known Clifford torus.

Given a surface of $E(\kappa, \tau)$, we denote by $C$ its angle function and $S$ the squared norm of its second fundamental form (see Section 2.3 for the definitions of $C$ and $S)$. Then as our main result, the rigidity theorem can be stated as follows.

Theorem 1.1. Let $\Phi: \Sigma \rightarrow E(\kappa, \tau)$ be a minimal immersion of a compact surface $\Sigma$. Then it holds the Simons' type integral inequality

$$
\int_{\Sigma}\left\{S^{2}-\left[2 \tau^{2}+\left(\kappa-4 \tau^{2}\right)\left(5 C^{2}-1\right)\right] S+2\left(\kappa-4 \tau^{2}\right) \tau^{2}\left(3 C^{2}-1\right)\right\} d \sigma \geq 0
$$

where the equality holds if and only if $\Phi$ is of parallel second fundamental form.

In particular, if $\Phi: \Sigma \rightarrow \mathbb{S}_{b}^{3}(\kappa, \tau)\left(\kappa \neq 4 \tau^{2}\right)$ is a minimal immersion of a compact surface $\Sigma$, then equality holds in (1.1) if and only if $\Phi: \Sigma \rightarrow \mathbb{S}_{b}^{3}(\kappa, \tau)$ is the Clifford torus. The latter case occurs only when $C \equiv 0$ and $S \equiv 2 \tau^{2}$.

Remark 1.2. It is well known that $S$, the squared norm of the second fundamental form of a compact minimally immersed surface $M$ in the unit sphere $\mathbb{S}^{3}(1)$, satisfies the Simons' integral inequality

$$
\int_{M} S(S-2) d \sigma \geq 0
$$

where the equality holds if and only if $M$ is either the equatorial sphere or the Clifford torus 77. Compared to the difference between (1.1) for $\mathbb{S}_{b}^{3}(\kappa, \tau)$ and (1.2) for $\mathbb{S}^{3}(1)$, it is remarkable that the equality sign in (1.1) is attainable only by the Clifford torus. This can be proven by the conclusion of Theorem 4.4 .

Remark 1.3. From the integral inequality (1.1), an interesting pointwise pinching result can be obtained in the case of Berger sphere $\mathbb{S}_{b}^{3}(\kappa, \tau)$ for $\kappa>4 \tau^{2}$. See Corollary 4.7 and Remark 4.8 . 


\section{Preliminaries}

\subsection{Homogeneous Riemannian 3-manifolds with 4-dimensional isometry}

group. We now recall from B. Daniel [9] some well-known facts for $E(\kappa, \tau)$.

Let $g$ be the Riemannian metric of $E(\kappa, \tau)$, which we also denote by $\langle\cdot, \cdot\rangle$. Denote by $\bar{\nabla}$ the Riemannian connection with respect to $g$. There exists a Riemannian submersion $\Pi: E(\kappa, \tau) \rightarrow \mathbb{M}^{2}(\kappa)$, where $\mathbb{M}^{2}(\kappa)$ is a 2-dimensional simply connected space form of constant curvature $\kappa$, with totally geodesic fibers, and there exists a unit Killing field $\xi$ on $E(\kappa, \tau)$ which is vertical with respect to $\Pi$. We will assume that $E(\kappa, \tau)$ is oriented, and then we can define a vectorial product $\times$, such that if $\left\{e_{1}, e_{2}\right\}$ are linearly independent vectors at a point $p$, then $\left\{e_{1}, e_{2}, e_{1} \times e_{2}\right\}$ is the orientation at $p$. Then the properties of $\xi$ imply (see [9]) that for any vector field $X$ on $E(\kappa, \tau)$ it holds the relation $\bar{\nabla}_{X} \xi=\tau X \times \xi$.

The Riemannian curvature tensor $\bar{R}$ of $E(\kappa, \tau)$ is defined by the following convention:

$$
\bar{R}(X, Y) Z=\bar{\nabla}_{Y} \bar{\nabla}_{X} Z-\bar{\nabla}_{X} \bar{\nabla}_{Y} Z+\bar{\nabla}_{[X, Y]} Z,
$$

where $X, Y, Z$ are tangent vector fields of $E(\kappa, \tau)$. Then we have

Proposition 2.1 (9]). For all vector fields $X, Y, Z, W$ on $E(\kappa, \tau)$ we have

$$
\langle\bar{R}(X, Y) Z, W\rangle=\left(\kappa-3 \tau^{2}\right)\left\langle R_{0}(X, Y) Z, W\right\rangle+\left(\kappa-4 \tau^{2}\right)\left\langle R_{1}(\xi ; X, Y) Z, W\right\rangle
$$

with

$$
\begin{gathered}
R_{0}(X, Y) Z=\langle X, Z\rangle Y-\langle Y, Z\rangle X \\
R_{1}(\xi ; X, Y) Z=\langle Y, \xi\rangle\langle Z, \xi\rangle X+\langle Y, Z\rangle\langle X, \xi\rangle \xi \\
\\
-\langle X, Z\rangle\langle Y, \xi\rangle \xi-\langle X, \xi\rangle\langle Z, \xi\rangle Y .
\end{gathered}
$$

It follows that the expression of the $(1,3)$ type Riemannian curvature tensor is

$$
\bar{R}(X, Y) Z=\left(\kappa-3 \tau^{2}\right) R_{0}(X, Y) Z+\left(\kappa-4 \tau^{2}\right) R_{1}(\xi ; X, Y) Z,
$$

where $X, Y, Z$ are vector fields on $E(\kappa, \tau)$.

2.2. Berger spheres $\mathbb{S}_{b}^{3}(\kappa, \tau)$. The Berger sphere is the special case of $E(\kappa, \tau)$ when $\kappa>0$ and $\tau \neq 0$. More precisely, a Berger sphere is a usual 3 -sphere $\mathbb{S}^{3}=\left\{(z, w) \in \mathbb{C}^{2}:|z|^{2}+|w|^{2}=1\right\}$ endowed with the metric $g=\langle,\rangle_{b}$ as that

$$
\langle X, Y\rangle_{b}=\frac{4}{\kappa}\left[\langle X, Y\rangle_{0}+\left(\frac{4 \tau^{2}}{\kappa}-1\right)\langle X, V\rangle_{0}\langle Y, V\rangle_{0}\right]
$$

where $\langle,\rangle_{0}$ stands for the usual metric on the sphere, $V_{(z, w)}:=(i z, i w)$ for each $(z, w) \in \mathbb{S}^{3}$ and $\kappa, \tau$ are real numbers with $\kappa>0$ and $\tau \neq 0$. The Berger sphere $\left(\mathbb{S}^{3},\langle,\rangle_{b}\right)$ as above will be denoted by $\mathbb{S}_{b}^{3}(\kappa, \tau)$.

The Hopf fibration $\Pi: \mathbb{S}_{b}^{3}(\kappa, \tau) \rightarrow \mathbb{S}^{2}(\kappa)$, where $\mathbb{S}^{2}(\kappa)$ stands for the 2-sphere of radius $1 / \sqrt{\kappa}$, defined by

$$
\Pi(z, w)=\frac{2}{\sqrt{\kappa}}\left(z \bar{w}, \frac{1}{2}\left(|z|^{2}-|w|^{2}\right)\right),
$$

is a Riemannian submersion whose fibers are geodesics. The vertical unit Killing vector field is given by $\xi=\frac{\kappa}{4 \tau} V$. 
2.3. Surfaces in $E(\kappa, \tau)$. Let $\Phi: \Sigma \rightarrow E(\kappa, \tau)$ be an immersion of oriented surface $\Sigma$ and $\nabla$ the induced Riemannian connection on $\Sigma$. Let $N$ be the unit normal field on $\Sigma, T$ the tangential component of $\xi, h$ and $A$ the second fundamental form and the shape operator of $\Phi$ associated with $N$, respectively. Then we have

$$
\begin{aligned}
& T=\xi-C N, \quad C=\langle\xi, N\rangle, \\
& \bar{\nabla}_{X} Y=\nabla_{X} Y+h(X, Y), \\
& A X=-\bar{\nabla}_{X} N, \quad\langle A X, Y\rangle=\langle h(X, Y), N\rangle,
\end{aligned}
$$

where $X, Y$ are tangent vector fields on $\Sigma$. We call $C$ the angle function of $\Sigma$.

Let $\left\{e_{1}, e_{2}, e_{3}=N\right\}$ be an orthonormal positively oriented moving frame of $E(\kappa, \tau)$ such that $e_{1}, e_{2}$ are tangential to $\Sigma$, and let $\left\{\omega_{1}, \omega_{2}, \omega_{3}\right\}$ be the coframe. Then the second fundamental form $h$ of $\Sigma$ has the expression

$$
h=\sum_{i, j=1}^{2} h_{i j} \omega_{i} \otimes \omega_{j} \otimes N,
$$

where the coefficients $h_{i j}=\left\langle h\left(e_{i}, e_{j}\right), N\right\rangle$.

The mean curvature $H$ and the squared norm $S$ of the second fundamental form $h$ of $\Sigma$ are defined as follows:

$$
H=\frac{1}{2}\left(h_{11}+h_{22}\right), \quad S=|h|^{2}=\sum_{i, j}\left(h_{i j}\right)^{2} .
$$

Let $\omega_{i j}$ be the connection forms associated with $\left\{e_{1}, e_{2}\right\}$, which are defined by

$$
d \omega_{i}=\sum_{j} \omega_{i j} \wedge \omega_{j}, \omega_{i j}+\omega_{j i}=0 .
$$

The first and second covariant derivatives $h_{i j k}$ and $h_{i j k l}$ of $h_{i j}$ are thus defined by

$$
\begin{gathered}
\sum_{k} h_{i j k} \omega_{k}=d h_{i j}+\sum_{k} h_{k j} \omega_{k i}+\sum_{k} h_{i k} \omega_{k j}, \\
\sum_{l} h_{i j k l} \omega_{l}=d h_{i j k}+\sum_{l} h_{l j k} \omega_{l i}+\sum_{l} h_{i l k} \omega_{l j}+\sum_{l} h_{i j l} \omega_{l k} .
\end{gathered}
$$

The Gauss equation of $\Phi: \Sigma \rightarrow E(\kappa, \tau)$ is (cf. [9])

$$
K=\operatorname{det} A+\tau^{2}+\left(\kappa-4 \tau^{2}\right) C^{2}=2 H^{2}-\frac{S}{2}+\tau^{2}+\left(\kappa-4 \tau^{2}\right) C^{2},
$$

where $K$ is the Gauss curvature of $\Sigma$, and $\operatorname{det} A=h_{11} h_{22}-\left(h_{12}\right)^{2}$.

The Codazzi equation of $\Phi: \Sigma \rightarrow E(\kappa, \tau)$ now reads as

$$
h_{i j k}-h_{i k j}=-\bar{R}_{3 i j k}, \quad 1 \leq i, j, k \leq 2,
$$

where

$$
\bar{R}_{3 i j k}=\bar{R}\left(N, e_{i}, e_{j}, e_{k}\right)=\left\langle\bar{R}\left(e_{k}, e_{j}\right) e_{i}, N\right\rangle .
$$

By using Proposition 2.1] we have

$$
\begin{gathered}
\left\langle R_{0}\left(e_{k}, e_{j}\right) e_{i}, N\right\rangle=0 \\
\left\langle R_{1}\left(\xi ; e_{k}, e_{j}\right) e_{i}, N\right\rangle=\left\langle\delta_{i j}\left\langle e_{k}, \xi\right\rangle \xi-\delta_{i k}\left\langle e_{j}, \xi\right\rangle \xi, N\right\rangle=C\left(\delta_{i j}\left\langle e_{k}, \xi\right\rangle-\delta_{i k}\left\langle e_{j}, \xi\right\rangle\right) .
\end{gathered}
$$

It follows that

$$
\bar{R}_{3 i j k}=C\left(\kappa-4 \tau^{2}\right)\left(\delta_{i j}\left\langle e_{k}, \xi\right\rangle-\delta_{i k}\left\langle e_{j}, \xi\right\rangle\right) .
$$


Denote $T=\sum_{i=1}^{2} T_{i} e_{i}$. Then the Codazzi equation becomes (cf. [9])

$$
h_{i j k}-h_{i k j}=C\left(\kappa-4 \tau^{2}\right)\left(\delta_{i k} T_{j}-\delta_{i j} T_{k}\right) .
$$

Let $R_{i j k l}$ be the components of the Riemannian curvature tensor $R$ of $\Sigma$, which satisfy

$$
-\frac{1}{2} \sum_{k, l} R_{i j k l} \omega_{k} \wedge \omega_{l}=d \omega_{i j}-\sum_{k} \omega_{i k} \wedge \omega_{k j} .
$$

Then we have the Ricci identity

$$
h_{i j k l}-h_{i j l k}=\sum_{m} h_{m j} R_{m i k l}+\sum_{m} h_{i m} R_{m j k l} .
$$

At any fixed point $p \in \Sigma$, we can suitably choose the local orthonormal frame field $\left\{e_{1}, e_{2}\right\}$ such that $h_{i j}(p)=\lambda_{i} \delta_{i j}$. If not stated otherwise, all calculations in sequel are carried out at $p$. This will be stated when it is necessary.

Denote $d C=\sum_{i} C_{i} \omega_{i}$. Then we have

$$
\begin{aligned}
C_{i} & =e_{i}\langle N, \xi\rangle=\left\langle\bar{\nabla}_{e_{i}} N, \xi\right\rangle+\left\langle N, \bar{\nabla}_{e_{i}} \xi\right\rangle \\
& =\left\langle-A e_{i}, \xi\right\rangle+\left\langle N, \tau e_{i} \times \xi\right\rangle=-\lambda_{i}\left\langle e_{i}, \xi\right\rangle+\tau\left\langle N \times e_{i}, \xi\right\rangle \\
& =-\lambda_{i} T_{i}+\tau\left\langle N \times e_{i}, \xi\right\rangle .
\end{aligned}
$$

We need to calculate $T_{i, j}$ for later use.

$$
\begin{aligned}
T_{i, j} & :=\nabla_{e_{j}}\left\langle T, e_{i}\right\rangle-\left\langle T, \nabla_{e_{j}} e_{i}\right\rangle=\nabla_{e_{j}}\left\langle\xi, e_{i}\right\rangle-\left\langle T, \nabla_{e_{j}} e_{i}\right\rangle \\
& =\left\langle\bar{\nabla}_{e_{j}} \xi, e_{i}\right\rangle+\left\langle\xi, \bar{\nabla}_{e_{j}} e_{i}\right\rangle-\left\langle T, \nabla_{e_{j}} e_{i}\right\rangle \\
& =\left\langle\tau e_{j} \times \xi, e_{i}\right\rangle+\left\langle\xi, h_{i j} N\right\rangle=h_{i j} C+\tau C \delta_{12}^{i j},
\end{aligned}
$$

where $\delta_{12}^{i j}$ is the generalized Kronecker symbols, i.e., $\delta_{12}^{12}=-\delta_{12}^{21}=1$ and $\delta_{12}^{11}=$ $\delta_{12}^{22}=0$.

Finally, for later purpose we look at the invariants of Hopf surfaces of $E(\kappa, \tau)(\tau \neq$ $0)$ in more detail.

2.4. Hopf surfaces in $E(\kappa, \tau)(\tau \neq 0)$. By definition, any Hopf surface in $E(\kappa, \tau)$ $(\tau \neq 0)$ has a vanishing angle function, i.e., $C=0$. Since it is flat, by (2.3) it also satisfies $S=4 H^{2}+2 \tau^{2}$. Here $H$ could be not a constant. Moreover, it was shown in [3] that surfaces in $E(\kappa, \tau)(\tau \neq 0)$ with parallel second fundamental form are Hopf cylinders. See Theorem 4.5

Next we consider the Clifford torus in $\mathbb{S}_{b}^{3}(\kappa, \tau)$. Recall that the Clifford torus has a parameterization $\phi: \mathbb{R}^{2} \rightarrow \mathbb{S}^{3}$ defined by

$$
\phi(s, t)=\left(\cos (s) e^{i t}, \sin (s) e^{i t}\right) .
$$

Put

$$
\phi_{s}=\left(-\sin (s) e^{i t}, \cos (s) e^{i t}\right), \quad \phi_{t}=\left(i \cos (s) e^{i t}, i \sin (s) e^{i t}\right) .
$$

Then we see that $V=\phi_{t}$ and $\xi=\frac{\kappa}{4 \tau} \phi_{t}$ is a unit tangent vector field of $\phi\left(\mathbb{R}^{2}\right)$. Thus $\phi$ is a Hopf torus. It is well known that the Clifford torus is a minimal surface in $\mathbb{S}_{b}^{3}(\kappa, \tau)$ and hence, by Gauss equation, it satisfies $S=2 \tau^{2}$. 


\section{Lemmas With Calculations on surfaces in $E(\kappa, \tau)$}

In this section, we make our main calculations on surfaces in $E(\kappa, \tau)$. We will begin with the following Simons' type formula, derived by using the Gauss-Codazzi equations and the Ricci identitiy.

Lemma 3.1. Let $\Phi: \Sigma \rightarrow E(\kappa, \tau)$ be an immersion of a surface $\Sigma$. Then, at $p \in \Sigma$, it holds that

$$
\begin{aligned}
\frac{1}{2} \Delta S=|\nabla h|^{2}+\sum_{i, k} \lambda_{i} h_{k k i i}+\left(\lambda_{1}-\lambda_{2}\right)^{2} K+2\left(\kappa-4 \tau^{2}\right) C^{2} S \\
-2\left(\kappa-4 \tau^{2}\right) \sum_{i} \lambda_{i}^{2} T_{i}^{2}+2\left(\kappa-4 \tau^{2}\right) \tau\left(\lambda_{1}-\lambda_{2}\right) T_{1} T_{2} \\
+2\left(\kappa-4 \tau^{2}\right) H \sum_{i} \lambda_{i} T_{i}^{2}-4\left(\kappa-4 \tau^{2}\right) H^{2} C^{2},
\end{aligned}
$$

where $\Delta$ denotes the Laplacian of the induced metric on $\Sigma$.

Moreover, if $\Phi$ is minimal, then at $p \in \Sigma$, it holds that

$$
\frac{1}{2} \Delta S=|\nabla h|^{2}+S\left[-S+2 \tau^{2}+\left(\kappa-4 \tau^{2}\right)\left(5 C^{2}-1\right)\right]+4\left(\kappa-4 \tau^{2}\right) \tau \lambda_{1} T_{1} T_{2} .
$$

Proof. By definition, we have

$$
\frac{1}{2} \Delta S=\sum_{i, j, k}\left(h_{i j k}\right)^{2}+\sum_{i, j, k} h_{i j} h_{i j k k} .
$$

Using the Codazzi equation (2.5) and the Ricci identity (2.6), we have

$$
\begin{aligned}
h_{i j k k}= & h_{k i j k}+\left(\kappa-4 \tau^{2}\right)\left[C\left(\delta_{i k} T_{j}-\delta_{i j} T_{k}\right)\right]_{, k} \\
= & h_{k i k j}+\sum_{m} h_{m i} R_{m k j k}+\sum_{m} h_{k m} R_{m i j k}+\left(\kappa-4 \tau^{2}\right)\left[C\left(\delta_{i k} T_{j}-\delta_{i j} T_{k}\right)\right]_{, k} \\
= & h_{k k i j}+\sum_{m} h_{m i} R_{m k j k}+\sum_{m} h_{k m} R_{m i j k} \\
& \quad+\left(\kappa-4 \tau^{2}\right)\left[C\left(T_{i}-\delta_{k i} T_{k}\right)\right]_{, j}+\left(\kappa-4 \tau^{2}\right)\left[C\left(\delta_{i k} T_{j}-\delta_{i j} T_{k}\right)\right]_{, k} .
\end{aligned}
$$

Thus,

$$
\begin{aligned}
\frac{1}{2} \Delta S= & |\nabla h|^{2}+\sum_{i, j, k} h_{i j} h_{k k i j}+\sum_{i, j, k, m} h_{i j} h_{m i} R_{m k j k}+\sum_{i, j, k, m} h_{i j} h_{k m} R_{m i j k} \\
& +\left(\kappa-4 \tau^{2}\right) \sum_{i, j, k}\left\{h_{i j}\left[C\left(T_{i}-\delta_{k i} T_{k}\right)\right]_{, j}+h_{i j}\left[C\left(\delta_{i k} T_{j}-\delta_{i j} T_{k}\right)\right]_{, k}\right\} .
\end{aligned}
$$

Denote

$$
\begin{aligned}
\mathrm{I} & :=\sum_{i, j, k, m} h_{i j} h_{m i} R_{m k j k}+\sum_{i, j, k, m} h_{i j} h_{k m} R_{m i j k}, \\
\mathrm{II} & :=\sum_{i, j, k} h_{i j}\left[C\left(T_{i}-\delta_{k i} T_{k}\right)\right]_{, j}+\sum_{i, j, k} h_{i j}\left[C\left(\delta_{i k} T_{j}-\delta_{i j} T_{k}\right)\right]_{, k} .
\end{aligned}
$$

It is obvious that

$$
\mathrm{I}=\sum_{i \neq k} h_{i i}^{2} R_{i k i k}+\sum_{i \neq k} h_{i i} h_{k k} R_{k i i k}=\left(\lambda_{1}-\lambda_{2}\right)^{2} K,
$$


where $K=R_{1212}$ is the Gauss curvature of $\Sigma$, and

$$
\begin{aligned}
\mathrm{II} & =\sum_{i, j} h_{i j}\left(C T_{i}\right)_{, j}+\sum_{i, j, k} h_{i j} C_{k}\left(\delta_{i k} T_{j}-\delta_{i j} T_{k}\right)+C \sum_{i, j, k} h_{i j}\left(\delta_{i k} T_{j, k}-\delta_{i j} T_{k, k}\right) \\
& =2 \sum_{i} \lambda_{i} C_{i} T_{i}+2 C \sum_{i} \lambda_{i} T_{i, i}-2 H \sum_{k} C_{k} T_{k}-2 C H \sum_{k} T_{k, k} .
\end{aligned}
$$

By using (2.7) and (2.8), we have

$$
\mathrm{II}=-2 \sum_{i} \lambda_{i}^{2} T_{i}^{2}+2 \tau\left(\lambda_{1}-\lambda_{2}\right) T_{1} T_{2}+2 C^{2} S+2 H \sum_{i} \lambda_{i} T_{i}^{2}-4 H^{2} C^{2}
$$

Putting (3.4) and (3.5) into (3.3), we get the assertion (3.1).

If $\Phi$ is minimal, then we have $\lambda_{2}=-\lambda_{1}$, and equation (3.1) reduces to

$$
\frac{1}{2} \Delta S=|\nabla h|^{2}+2 K S+2\left(\kappa-4 \tau^{2}\right) C^{2} S-\left(\kappa-4 \tau^{2}\right)|T|^{2} S+4\left(\kappa-4 \tau^{2}\right) \lambda_{1} \tau T_{1} T_{2},
$$

which together with (2.3) yields (3.2).

We will need to know the divergence of $\nabla_{T} T$, stated by $\mathrm{F}$. Torralbo and $\mathrm{F}$. Urbano in [18, omitting computation details. Here, for reader's convenience, we present it in a slightly more detailed way.

Lemma $3.2([18)$. Let $\Phi: \Sigma \rightarrow E(\kappa, \tau)$ be an immersion of a surface $\Sigma$. Then it holds on $\Sigma$ that

$$
\operatorname{div}\left(\nabla_{T} T\right)=K|T|^{2}+\langle\nabla \operatorname{div}(T), T\rangle+|\nabla T|^{2}-4 \tau^{2} C^{2},
$$

where $T$ is the tangential component of $\xi$ and $C$ is the angle function of $\Sigma$.

Moreover, if $\Phi$ is minimal, then it holds on $\Sigma$ that

$$
\operatorname{div}\left(\nabla_{T} T\right)=K|T|^{2}+\left(S-2 \tau^{2}\right) C^{2} .
$$

Proof. Around each point of $\Sigma$, we choose an arbitrary local orthonormal frame $\left\{e_{1}, e_{2}\right\}$. Then, by definition, direct computation shows that

$$
\begin{aligned}
\operatorname{div}\left(\nabla_{T} T\right) & =\sum_{i, j}\left\langle\nabla_{e_{i}}\left(T_{j} \nabla_{e_{j}} T\right), e_{i}\right\rangle \\
& =\sum_{i, j, k}\left\langle\nabla_{e_{i}}\left(T_{j} T_{k, j} e_{k}\right), e_{i}\right\rangle \\
& =\sum_{i, j, k}\left\langle\left(T_{j} T_{k, j}\right)_{, i} e_{k}, e_{i}\right\rangle \\
& =\sum_{i, j}\left(T_{j} T_{i, j}\right)_{, i} \\
& =\sum_{i, j} T_{j, i} T_{i, j}+\sum_{i, j} T_{j} T_{i, j i} .
\end{aligned}
$$

According to (2.8), we can get

$$
\begin{aligned}
\sum_{i, j} T_{j, i} T_{i, j} & =\sum_{i, j}\left(T_{i, j}\right)^{2}-\left(T_{1,2}\right)^{2}-\left(T_{2,1}\right)^{2}+2 T_{1,2} T_{2,1} \\
& =\sum_{i, j}\left(T_{i, j}\right)^{2}-4 \tau^{2} C^{2}=|\nabla T|^{2}-4 \tau^{2} C^{2}
\end{aligned}
$$


and, by the Ricci identity, we have

$$
\begin{aligned}
\sum_{i, j} T_{j} T_{i, j i} & =\sum_{i, j} T_{j}\left(T_{i, i j}+T_{k} R_{k i j i}\right) \\
& =\langle\nabla \operatorname{div}(T), T\rangle+\sum_{i, j}\left(T_{j}\right)^{2} R_{j i j i} \\
& =\langle\nabla \operatorname{div}(T), T\rangle+K|T|^{2} .
\end{aligned}
$$

Putting (3.9) and (3.10) into (3.8), we get (3.6).

If $\Phi$ is minimal, then according to (2.8), we have

$$
|\nabla T|^{2}=\sum_{i, j}\left(T_{i, j}\right)^{2}=C^{2} S+2 \tau^{2} C^{2}
$$

and the divergence of $T$ satisfies

$$
\operatorname{div} T=\sum_{i} T_{i, i}=2 H C=0 .
$$

Putting (3.11) and (3.12) into (3.6), we get (3.7).

We also need the calculation for $\Delta|T|^{2}$.

Lemma 3.3. Let $\Phi: \Sigma \rightarrow E(\kappa, \tau)$ be an immersion of a surface $\Sigma$. Then, at $p \in \Sigma$, it holds that

$$
\begin{gathered}
\frac{1}{2} \Delta|T|^{2}=K|T|^{2}+\langle\nabla \operatorname{div}(T), T\rangle+|\nabla T|^{2}+2 \tau^{2}\left(C^{2}-1\right) \\
+2 \tau\left(\lambda_{1}-\lambda_{2}\right) T_{1} T_{2} .
\end{gathered}
$$

Moreover, if $\Phi$ is minimal, it holds at $p \in \Sigma$ that

$$
\frac{1}{2} \Delta|T|^{2}=K|T|^{2}+C^{2} S+2 \tau^{2}\left(2 C^{2}-1\right)+4 \tau \lambda_{1} T_{1} T_{2} .
$$

Proof. By direct calculation, with the use of Ricci identity and (2.8), we have

$$
\begin{aligned}
\frac{1}{2} \Delta|T|^{2} & =\sum_{i, j}\left(T_{i, j}\right)^{2}+\sum_{i, j} T_{i} T_{i, j j} \\
& =|\nabla T|^{2}+\sum_{i, j} T_{i}\left(T_{j, i}+2 \tau C \delta_{12}^{i j}\right)_{, j} \\
& =|\nabla T|^{2}+\sum_{i, j} T_{i} T_{j, i j}+2 \tau \sum_{i, j} T_{i} C_{j} \delta_{12}^{i j} \\
& =K|T|^{2}+\langle\nabla \operatorname{div}(T), T\rangle+|\nabla T|^{2}+2 \tau \sum_{i, j} T_{i} C_{j} \delta_{12}^{i j} .
\end{aligned}
$$

On the other hand, (2.7) further implies that

$$
\begin{aligned}
\sum_{i, j} T_{i} C_{j} \delta_{12}^{i j} & =T_{1} C_{2}-T_{2} C_{1} \\
& =T_{1}\left(-\lambda_{2} T_{2}+\tau\left\langle N \wedge e_{2}, \xi\right\rangle\right)-T_{2}\left(-\lambda_{1} T_{1}+\tau\left\langle N \wedge e_{1}, \xi\right\rangle\right) \\
& =\left(\lambda_{1}-\lambda_{2}\right) T_{1} T_{2}-\tau T_{1}^{2}-\tau T_{2}^{2} \\
& =\left(\lambda_{1}-\lambda_{2}\right) T_{1} T_{2}+\tau\left(C^{2}-1\right) .
\end{aligned}
$$

Putting (3.16) into (3.15), the assertion (3.13) follows.

If $\Phi$ is minimal, by inserting (3.11) and (3.12) into (3.13), we immediately get (3.14). 
Now we can state the main results of this section, which will be crucial for the proof of Theorem 1.1 .

Lemma 3.4. Let $\Phi: \Sigma \rightarrow E(\kappa, \tau)$ be a minimal immersion of a surface $\Sigma$. Then it holds on $\Sigma$ that

$$
\begin{aligned}
& \frac{1}{2} \Delta S-\left(\kappa-4 \tau^{2}\right)\left[\frac{1}{2} \Delta|T|^{2}-\operatorname{div}\left(\nabla_{T} T\right)\right] \\
& =|\nabla h|^{2}-S^{2}+\left[2 \tau^{2}+\left(\kappa-4 \tau^{2}\right)\left(5 C^{2}-1\right)\right] S-2\left(\kappa-4 \tau^{2}\right) \tau^{2}\left(3 C^{2}-1\right) .
\end{aligned}
$$

Proof. According to Lemmas 3.1, 3.2 and 3.3, we see that (3.17) holds at $p \in \Sigma$. The assertion finally follows from the fact that the right hand of (3.17) is independent of the choice of the orthonormal frame.

\section{BASIC FACTS AND PROOF OF THE RIGIDITY THEOREMS}

We begin with a simple observation.

Proposition 4.1. Let $\Phi: \Sigma \rightarrow E(\kappa, \tau)(\tau \neq 0)$ be a minimal immersion of a compact surface $\Sigma$. Then the angle function $C \equiv 0$ is equivalent to the fact that the squared norm of the second fundamental form of $\Phi$ satisfies $S \equiv 2 \tau^{2}$.

Proof. If $C \equiv 0$, then the Gauss equation (2.3) and the assumption $H \equiv 0$ imply that

$$
K=\operatorname{det} A+\tau^{2}+\left(\kappa-4 \tau^{2}\right) C^{2}=-\frac{S}{2}+\tau^{2} .
$$

On the other hand, $C \equiv 0$, showing that $\xi$ is a tangent vector field of $\Sigma$. Thus, for a tangent vector field $v, \bar{\nabla}_{v} \xi=\tau v \times \xi \in T^{\perp} \Sigma$. This implies that $\nabla_{v} \xi=0$ and therefore $R(v, \xi, \xi, v)=0$. Hence we have $K \equiv 0$, and then $S \equiv 2 \tau^{2}$ follows.

The converse follows from the integral formula (3.3) in 18 . We write it out in more detail. If $S \equiv 2 \tau^{2}$, then the Gauss equation (2.3) gives that $K=\left(\kappa-4 \tau^{2}\right) C^{2}$. By (3.7), we obtain

$$
\operatorname{div}\left(\nabla_{T} T\right)=\left(\kappa-4 \tau^{2}\right) C^{2}\left(1-C^{2}\right) .
$$

The divergence theorem then gives that

$$
\int_{\Sigma} C^{2}\left(1-C^{2}\right) d \sigma=0
$$

Therefore, $C^{2}\left(1-C^{2}\right)=0$ on $\Sigma$. The following remark shows that $C^{2}=1$ does not occur, so we get $C=0$ on $\Sigma$ as claimed.

Remark 4.2. As pointed out in [18], if $C^{2}=1$, then it is well known that $\tau=0$ and the surface is a totally geodesic slice of $M^{2}(\kappa) \times \mathbb{R}$. This holds true even without the compactness assumption.

Remark 4.3. We also note that, surfaces in $E(\kappa, \tau)(\tau \neq 0)$ with $C \equiv 0$ are Hopf cylinders, and it can be checked directly that they have parallel second fundamental form. Indeed, if $C \equiv 0$, then the surface is $\Pi^{-1}(\gamma)$ for some curve $\gamma$, and by a symmetry argument (or an easy computation) we immediately get that $\gamma$ is a geodesic. Related to this fact, see also Theorem 4.5.

We next recall that, in contrast to the fact that there exists totally umbilic surfaces in the round sphere, it was proved by R. Souam and E. Toubiana [15] that in $E(\kappa, \tau)(\tau \neq 0)$ there does not exist any totally umbilic surfaces. 
Theorem 4.4 ([15]). There exist no totally umbilic surfaces (even non-complete) in the 3 -manifolds $E(\kappa, \tau)$ with $\tau \neq 0$. In particular, there are no totally geodesic surfaces in $E(\kappa, \tau)$ with $\tau \neq 0$.

On the other hand, surfaces with parallel second fundamental form in BianchiCartan-Vranceanu spaces are shown to be the Hopf cylinders by M. Belkhelfa, F. Dillen, and J. Inoguchi in [3. Here, for $E(\kappa, \tau)(\tau \neq 0)$, we include a shorter proof.

Theorem 4.5 (cf. Theorem 8.2 of [3]). Let $\Phi: \Sigma \rightarrow E(\kappa, \tau)(\tau \neq 0)$ be a surface with parallel second fundamental form. Then $\Phi$ is a Hopf cylinder.

Proof. As $\nabla h \equiv 0$, the Codazzi equation (2.5) becomes

$$
\left(\kappa-4 \tau^{2}\right) C\left(\delta_{i k} T_{j}-\delta_{i j} T_{k}\right)=0 .
$$

As $\kappa \neq 4 \tau^{2}$, we get

$$
C T_{j}=0, \quad \forall j=1,2 .
$$

This implies that we have either $C=0$ or $T=0$ on $\Sigma$.

If $T=0$ holds on $\Sigma$, then $C^{2} \equiv 1$ and according to Remark 4.2 it implies that $\tau=0$, a contradiction. Hence it holds that $C \equiv 0$. Namely, $\Phi$ is a Hopf cylinder.

Proof of Theorem 1.1. Integrating (3.17) over $\Sigma$ and using the divergence theorem we immediately obtain the first assertion. Then, the second assertion is a direct consequence of Theorem 4.5 and a fact that the Clifford torus is the only minimal Hopf torus in $\mathbb{S}_{b}^{3}(\kappa, \tau)$.

Remark 4.6. The important fact that the Clifford torus is the only minimal Hopf torus in $\mathbb{S}_{b}^{3}(\kappa, \tau)$ is referred to in the third section of [2]. From [2], we know that a similar fact also holds for a Hopf torus in the round sphere. In fact, this latter fact can be derived directly from Pinkall's computation for the mean curvature of a Hopf torus 14 .

In contrast with the above fact, an interesting phenomenon worth noting is that in [18, it was shown that the only flat compact surfaces in $\mathbb{S}_{b}^{3}(\kappa, \tau)$ are the Hopf tori. This result in the Berger sphere contrasts with the case of the round sphere where, besides the Hopf tori, there are other flat tori (see [14,21]).

To get more explicit information from Theorem 1.1, we next consider the Berger sphere $\mathbb{S}_{b}^{3}(\kappa, \tau)$ with $\kappa>4 \tau^{2}$. For such cases the following equation on $x$,

$$
x^{2}-\left[2 \tau^{2}+\left(\kappa-4 \tau^{2}\right)\left(5 C^{2}-1\right)\right] x+2\left(\kappa-4 \tau^{2}\right) \tau^{2}\left(3 C^{2}-1\right)=0,
$$

has two distinct real number solutions $x_{1}=a(\kappa, \tau, C)$ and $x_{2}=b(\kappa, \tau, C)$, where

$$
\begin{aligned}
& a(\kappa, \tau, C)=\frac{1}{2}\left[2 \tau^{2}+\left(\kappa-4 \tau^{2}\right)\left(5 C^{2}-1\right)-\sqrt{\rho}\right], \\
& b(\kappa, \tau, C)=\frac{1}{2}\left[2 \tau^{2}+\left(\kappa-4 \tau^{2}\right)\left(5 C^{2}-1\right)+\sqrt{\rho}\right],
\end{aligned}
$$

and $\rho$ is defined by

$$
\rho=4 \tau^{4}+\left(\kappa-4 \tau^{2}\right)^{2}\left(5 C^{2}-1\right)^{2}+4\left(\kappa-4 \tau^{2}\right) \tau^{2}\left(1-C^{2}\right) .
$$


Obviously, $b(\kappa, \tau, C)$ is positive, whereas $a(\kappa, \tau, C)$ could be negative. Moreover, we have the relation $a(\kappa, \tau, C) \leq \tau^{2}<2 \tau^{2} \leq b(\kappa, \tau, C)$. Hence, as immediate consequences of Theorem 1.1, we have the following

Corollary 4.7. Let $\Phi: \Sigma \rightarrow \mathbb{S}_{b}^{3}(\kappa, \tau)\left(\kappa>4 \tau^{2}\right)$ be a minimal immersion of a compact surface $\Sigma$. If the squared norm $S$ of the second fundamental form of $\Phi$ satisfies $a(\kappa, \tau, C) \leq S \leq b(\kappa, \tau, C)$, then $C \equiv 0, S \equiv 2 \tau^{2}$ and $\Phi$ is the Clifford torus. More specifically, if $\tau^{2} \leq S \leq 2 \tau^{2}$, then $C \equiv 0, S \equiv 2 \tau^{2}$ and $\Phi$ is the Clifford torus.

Remark 4.8. Corollary 4.7 is considered by imposing the restriction $\kappa>4 \tau^{2}$, which obviously can be weakened. However, as the problem of establishing a similar pinching result as Corollary 4.7 for the case $\kappa<4 \tau^{2}$ is very interesting, it is still open for us.

Remark 4.9. For compact minimal surfaces in $\mathbb{S}_{b}^{3}(\kappa, \tau)$, a satisfactory pinching result might be like that if $0 \leq S \leq 2 \tau^{2}$, then $S \equiv 2 \tau^{2}$ and the surface is the Clifford torus. But, this is still an open problem for us.

Finally, motivated by Theorem 3.6 of [18, a similar rigidity result in terms of the squared norm of the second fundamental form can be derived as follows.

Proposition 4.10. Let $\Phi: \Sigma \rightarrow E(\kappa, \tau)$ be an immersion of a compact surface $\Sigma$.

(i) For $\kappa<4 \tau^{2}$, if $S \geq 4 H^{2}+2 \tau^{2}$ and $C^{2} \neq 1$, then $S \equiv 4 H^{2}+2 \tau^{2}$ and $\Phi$ is a Hopf torus. Consequently, a compact minimal surface of $\mathbb{S}_{b}^{3}(\kappa, \tau)\left(\kappa<4 \tau^{2}\right)$ with $S \geq 2 \tau^{2}$ and $C^{2} \neq 1$ is the Clifford torus with $S \equiv 2 \tau^{2}$.

(ii) For $\kappa>4 \tau^{2}$, if $S \leq 4 H^{2}+2 \tau^{2}$ and $C^{2} \neq 1$, then $S \equiv 4 H^{2}+2 \tau^{2}$ and $\Phi$ is a Hopf torus. Consequently, a compact minimal surface of $\mathbb{S}_{b}^{3}(\kappa, \tau)\left(\kappa>4 \tau^{2}\right)$ with $S \leq 2 \tau^{2}$ and $C^{2} \neq 1$ is the Clifford torus with $S \equiv 2 \tau^{2}$.

Proof. (i) We consider the first part. As $C^{2} \neq 1$, the tangential component $X$ of the Killing field $\xi$ is a vector field on $\Sigma$ without singular point. We conclude that the Euler-Poincaré characteristic $\chi(\Sigma)=0$ from the Hopf theorem. By Gauss-Bonnet theorem we get $\int_{\Sigma} K d \sigma=0$.

On the other hand, as $S \geq 4 H^{2}+2 \tau^{2}$, the Gauss equation implies that

$$
\begin{aligned}
K & =2 H^{2}-\frac{S}{2}+\tau^{2}+\left(\kappa-4 \tau^{2}\right) C^{2} \\
& \leq 2 H^{2}-\frac{S}{2}+\tau^{2} \\
& \leq 0 .
\end{aligned}
$$

Hence, we have $K=0$ and $S \equiv 4 H^{2}+2 \tau^{2}$ on $\Sigma$. The assertion then follows because from [18] we know that the only flat compact surfaces in $E(\kappa, \tau)$ are the Hopf tori.

(ii) This is similar to the proof of case (i).

Remark 4.11. As pointed out by the referee, Proposition 4.10 could be viewed as a direct consequence of Theorem 3.6 of [18.

\section{ACKNOWLEDGEMENTS}

The first-named author would like to thank Profs. Martin Kilian, Haizhong $\mathrm{Li}$, and Luc Vrancken for helpful discussions on a related topic. Furthermore, the authors would like to express their deep thanks to the referee the many comments and valuable proposals that helped to improve the paper. 


\section{REFERENCES}

[1] Uwe Abresch and Harold Rosenberg, A Hopf differential for constant mean curvature surfaces in $\mathbf{S}^{2} \times \mathbf{R}$ and $\mathbf{H}^{2} \times \mathbf{R}$, Acta Math. 193 (2004), no. 2, 141-174, DOI 10.1007/BF02392562. MR2134864(2006h:53003)

[2] Manuel Barros and Angel Ferrández, Willmore energy estimates in conformal Berger spheres, Chaos Solitons Fractals 44 (2011), no. 7, 515-521, DOI 10.1016/j.chaos.2011.04.003. MR.2813029

[3] Mohamed Belkhelfa, Franki Dillen, and Jun-Ichi Inoguchi, Surfaces with parallel second fundamental form in Bianchi-Cartan-Vranceanu spaces, PDEs, submanifolds and affine differential geometry (Warsaw, 2000), Banach Center Publ., vol. 57, Polish Acad. Sci. Inst. Math., Warsaw, 2002, pp. 67-87, DOI 10.4064/bc57-0-5. MR1972463(2004b:53103)

[4] L. Bianchi, Lezioni di Geometrie Differenziale (Italian), E. Spoerri Librao-Editore, 1894.

[5] L. Bianchi, Lezioni sulla teoria dei gruppi continui e finiti di transformazioni (Italian), Ed. Zanichelli, 1928.

[6] É. Cartan, Leçons sur la Géométrie des Espaces de Riemann (French), Gauthier-Villars, Paris, 1946. 2d ed. MR0020842 (8,602g)

[7] S. S. Chern, M. do Carmo, and S. Kobayashi, Minimal submanifolds of a sphere with second fundamental form of constant length, Functional Analysis and Related Fields (Proc. Conf. for M. Stone, Univ. Chicago, Chicago, Ill., 1968), Springer, New York, 1970, pp. 59-75. MR0273546(42 \#8424)

[8] Benoît Daniel, Isometric immersions into $\mathbb{S}^{n} \times \mathbb{R}$ and $\mathbb{H}^{n} \times \mathbb{R}$ and applications to minimal surfaces, Trans. Amer. Math. Soc. 361 (2009), no. 12, 6255-6282, DOI 10.1090/S0002-994709-04555-3. MR 2538594 (2010g:53107)

[9] Benoît Daniel, Isometric immersions into 3-dimensional homogeneous manifolds, Comment. Math. Helv. 82 (2007), no. 1, 87-131, DOI 10.4171/CMH/86. MR2296059 (2008a:53058)

[10] Isabel Fernández and Pablo Mira, A characterization of constant mean curvature surfaces in homogeneous 3-manifolds, Differential Geom. Appl. 25 (2007), no. 3, 281-289, DOI 10.1016/j.difgeo.2006.11.006. MR.2330457(2008e:53012)

[11] Jun-ichi Inoguchi and Joeri Van der Veken, A complete classification of parallel surfaces in three-dimensional homogeneous spaces, Geom. Dedicata 131 (2008), 159-172, DOI 10.1007/s10711-007-9222-0. MR:2369197 (2008m:53132)

[12] Shoshichi Kobayashi, Transformation groups in differential geometry, Springer-Verlag, New York, 1972. Ergebnisse der Mathematik und ihrer Grenzgebiete, Band 70. MR0355886 (50 \#8360)

[13] William H. Meeks III and Joaquín Pérez, Constant mean curvature surfaces in metric Lie groups, Geometric analysis: partial differential equations and surfaces, Contemp. Math., vol. 570, Amer. Math. Soc., Providence, RI, 2012, pp. 25-110, DOI 10.1090/conm/570/11304. MR.2963596

[14] U. Pinkall, Hopf tori in $S^{3}$, Invent. Math. 81 (1985), no. 2, 379-386, DOI 10.1007/BF01389060. MR799274 (86k:53075)

[15] Rabah Souam and Eric Toubiana, Totally umbilic surfaces in homogeneous 3-manifolds, Comment. Math. Helv. 84 (2009), no. 3, 673-704, DOI 10.4171/CMH/177. MR2507258 (2010f:53079)

[16] Francisco Torralbo, Compact minimal surfaces in the Berger spheres, Ann. Global Anal. Geom. 41 (2012), no. 4, 391-405. MR2897028

[17] Francisco Torralbo, Rotationally invariant constant mean curvature surfaces in homogeneous 3-manifolds, Differential Geom. Appl. 28 (2010), no. 5, 593-607, DOI 10.1016/j.difgeo.2010.04.007. MR2670089 (2011f:53137)

[18] Francisco Torralbo and Francisco Urbano, On the Gauss curvature of compact surfaces in homogeneous 3-manifolds, Proc. Amer. Math. Soc. 138 (2010), no. 7, 2561-2567, DOI 10.1090/S0002-9939-10-10316-5. MR2607886 (2011c:53142)

[19] Francisco Torralbo and Francisco Urbano, Compact stable constant mean curvature surfaces in homogeneous 3-manifolds, Indiana Univ. Math. J. 61 (2012), no. 3, 1129-1156, DOI 10.1512/iumj.2012.61.4667. MR3071695 
[20] G. Vranceanu, Leçons de Géométrie Différentielle. Vol. 1. Congruences. Formes de Pfaff. Groupes continus. Invariants et équivalence. Espaces à connexion affine. Espaces de Riemann. Espaces à connexion projective (French), Bucarest, 1947. MR0024671 (9,532c)

[21] Joel L. Weiner, Flat tori in $S^{3}$ and their Gauss maps, Proc. London Math. Soc. (3) 62 (1991), no. 1, 54-76, DOI 10.1112/plms/s3-62.1.54. MR.1078213(92d:53057)

School of Mathematics and Statistics, Zhengzhou University, Zhengzhou 450001, People's Republic of ChinA

E-mail address: huzj@zzu.edu.cn

School of Mathematics and Statistics, Zhengzhou University, Zhengzhou 450001, People's Republic of ChinA

E-mail address: dongliang040@sina.com

School of Mathematics and Statistics, Zhengzhou University, Zhengzhou 450001, People's Republic of China

Current address: School of Mathematics and Statistics, Beijing Institute of Technology, Beijing 102400, People's Republic of China

E-mail address: wangjingzzumath@163.com 